\title{
Proposta de Redesign da Plataforma Poesia Compilada acessível para pessoas com deficiência visual baixa visão para a difusão do Pensamento Computacional
}

\author{
Soraya R. S. Medeiros ${ }^{1}$, Humberto Rabelo ${ }^{1}$, Angélica F. Medeiros ${ }^{1}$, Cristóvão S. \\ Lima ${ }^{1}$, Hélio E. M. Júnior ${ }^{1}$, Janailton G. Pereira ${ }^{1}$, Mikaelly S. M. Nunes ${ }^{1}$, Tânia C. \\ M. Garcia ${ }^{2}$ \\ ${ }^{1}$ Departamento de Computação e Tecnologia \\ Universidade Federal do Rio Grande do Norte (UFRN) - Caicó, RN - Brasil \\ ${ }^{2}$ Departamento de Educação \\ Universidade Federal do Rio Grande do Norte (UFRN) - Caicó, RN - Brasil \\ \{soraya.roberta.js, hrabeloufrn, angelicafelixx, cristovao.s.lima, \\ heliojunior53\} @gmail.com, \{janailton.gp, mikaelly_sandy\}@hotmail.com, \\ tania_cristina2005@yahoo.com.br
}

\begin{abstract}
The quality of digital resources application in educational contexts is an important subject in academic environment. However, this environment tends to have usability, accessibility and communicability issues. This study proposes a redesign of Poesia Compilada platform for disseminating accessible teaching of Computational Thinking (PC) to people with low vision. It was divided in three steps: i) literacy review; ii) analysis of previous interface and iii) platform redesign. Redesign was implemented, including a menu with platform's main functions, a button to enter into Poesia editor, so as contrast and font size settings for easing use for low vision users, and a repository of poetries.
\end{abstract}

Resumo. A qualidade da aplicação de recursos digitais em contextos educacionais é uma temática importante para o meio acadêmico, porém, esses espaços tendem a apresentar problemas de usabilidade, acessibilidade e comunicabilidade. Este estudo objetiva propor o redesign da Plataforma Poesia Compilada para a difusão do ensino de Pensamento Computacional acessível para pessoas com deficiência visual baixa visão. Foi dividido em três etapas: i) revisão literária; ii) análise da interface anterior e iii) redesign da plataforma. Como resultados, implementou-se o redesign, com destaque para um menu com botões de contraste e aumento do tamanho de fonte para facilitar a utilização por usuários com baixa visão, bem como um repositório para as poesias.

\section{Introdução}

O estudo e a aplicação de recursos digitais em contextos educacionais é uma temática de grande importância para a sociedade e para o meio acadêmico, entretanto, ainda há uma grande lacuna no que diz respeito à usabilidade, comunicabilidade e acessibilidade 
VIII Congresso Brasileiro de Informática na Educação (CBIE 2019)

Anais do XXX Simpósio Brasileiro de Informática na Educação (SBIE 2019)

desses recursos, pois muitas pessoas têm necessidades específicas que nem sempre são atendidas Moita et al. (2017). Essas lacunas, inerentes ao design de tecnologias educacionais podem aumentar as dificuldades ou causar efeitos indesejados no usuário, como incômodos, distrações ou aversão por seu uso, impossibilitando a eficácia da proposta pedagógica da aplicação Barbosa and Silva (2010), Matos (2013) e Rosa and Matos (2015). Neste contexto, Carvalho and Duduchi (2009) apontam a necessidade de redesign dessas interfaces tendo como foco potencializar a experiência do usuário, propiciando interface funcional, fácil de ser aprendida e com poucas taxas de erros.

O redesign tem como intuito repensar e apontar problemas de interface e/ou interação para, a partir disto, desenvolver uma versão otimizada, seja sob o aspecto funcional ou visual Campos (2017). Além destes aspectos, Preece (2005) enfatiza a necessidade dos processos associados à cognição no processo de concepção das interfaces, visto que é papel do designer preparar projetos eficientes para que a aprendizagem dos usuários seja efetiva. Atrelada a esses fatores, nos último anos, vem se observando a produção de estudos envolvendo Pensamento Computacional (PC), que diz respeito à aplicação de conceitos da Ciência da Computação para a resolução de problemas cotidianos Wing (2006), utilizando ferramentas para proporcionar que crianças consigam aprender de forma lúdica.

Contudo, os trabalhos sobre o estado da arte de PC Ortiz and Pereira (2018), Araújo, Andrade and Guerrero (2016) permitem concluir que os objetivos se restringem a identificarem e relatarem as práticas, citarem as ferramentas utilizadas e os tipos de avaliações utilizadas, não se discutindo acerca do desenvolvimento ou do redesign de ferramentas para esses casos, com o foco em Interação Humano-Computador (IHC), o que delimita e exclui a compreensão sobre em que essas ferramentas estão se fundamentando para proporcionarem uma boa experiência ao usuário e, em consequência, atingir com o propósito pedagógico da atividade. Tendo como base essas problemáticas, este trabalho tem como objetivo propor o redesign da plataforma Poesia Compilada para a difusão do ensino de PC acessível para pessoas com deficiência visual baixa-visão. Este estudo é resultado parcial de um Projeto de Pesquisa maior que objetiva difundir o PC, utilizando a abordagem ensino de algoritmos para alunos do $5^{\circ} \mathrm{e}$ $6^{\circ}$ ano do Ensino Fundamental, por meio da criação de poesias compiladas.

Composto por uma página HTML simples, utilizando Javascript e CSS, o sistema se propõe a servir como um espaço para as pessoas terem acesso e replicarem a ideia do Manifesto Literário Poesia Compilada por meio da escrita de poemas no editor Poesia(). Além disso, artigos publicados relatam que a primeira versão do software foi disponibilizada em 2015. Contudo, essa versão apresenta várias limitações, como a inexistência da execução da função exportar a poesia em navegadores, como o Google Chrome, a falta da aplicação dos critérios a acessibilidade e comunicabilidade, e a ausência de um ambiente para salvar as poesias. Por esses motivos, esta nova versão buscou elencar novos requisitos e propor um designer que levasse em consideração os critérios de IHC, proporcionando, dentre outras melhorias, o suporte para usuários com deficiência visual baixa-visão, ampliando o público que terá acesso ao conteúdo.

Esclarecidos a contextualização e justificativa do trabalho, apresenta-se a estrutura do presente artigo, que está organizado da seguinte forma: 2. Conceitos relacionados à $(\mathrm{Re})$ Design de Interfaces Educacionais; 3. Alguns trabalhos relacionados 
VIII Congresso Brasileiro de Informática na Educação (CBIE 2019)

Anais do XXX Simpósio Brasileiro de Informática na Educação (SBIE 2019)

à temática descrita; 4. Descreve e detalha a metodologia utilizada; 5. Apresenta a solução proposta, os resultados e discussões; 6. Por fim, contribuições esperadas, com considerações finais e os trabalhos futuros.

\section{2. (Re)design de Interfaces Educacionais}

Para Rocha and Baranauskas (2003), a Interação Humano-Computador (IHC) trabalha com o design de sistemas computacionais tendo como objetivo auxiliar os usuários a conseguirem executar suas atividades de maneira segura e eficiente. Já na visão de Barbosa and Silva (2010), a IHC é compreendida como uma área que estuda o ser humano e suas interações com os computadores. Além disso, para entender os fenômenos envolvidos no uso de sistemas computacionais interativos, ela faz uso do conhecimento de diversas outras áreas, tais como Psicologia, Linguística, Ergonomia, Design e Semiótica. A autora reforça que além desses fatores, um outro aspecto discutido em IHC é a Qualidade. Para que ela ocorra é necessário que se obedeça a alguns critérios: usabilidade, experiência do usuário, acessibilidade e comunicabilidade.

Seguindo tais pensamentos, percebe-se que a utilização de IHC é prioritária para se estabelecer um ambiente inclusivo e acessível dentro de uma plataforma e demais tecnologias educacionais. Sendo importante destacar, ainda, que usabilidade e acessibilidade é uma significante combinação para possibilitar a inclusão. Neste sentido, Barros (2017), defende que o uso de tecnologias que permitem a inclusão são capazes de promover meios que despertam no discente a capacidade de desafiar-se, experimentar e conhecer, desenvolvendo a construção individual e coletiva de novos conhecimentos. Todavia, para que isso seja bem implementado, é necessário que exista um ator responsável por preparar e definir as etapas em que os critérios de IHC serão utilizados. Segundo Preece (2005), compreender os processos associados à cognição é relevante ao processo de design das interfaces, visto que é papel do designer preparar projetos eficientes para que de fato a aprendizagem dos usuários seja efetiva. O designer deve considerar princípios e diretrizes adequadas a cada situação acerca do domínio do problema, dos usuários e das suas atividades. O Quadro 1 apresenta alguns tipos de processos cognitivos e suas descrições, além de implicações do design associados que, uma vez adotadas, podem facilitar seu atingimento. Ciente dessas necessidades, cabe apontar que o projeto de interface deve relacionar os critérios de IHC com o processo de desenvolvimento da interface, pois enquanto o projeto permite dar uma visão ampla sobre o que vai ser elaborado, o processo prepara e define as etapas para que elas sejam realizadas.

Quadro 1. Processos cognitivos segundo Preece (2005)

\begin{tabular}{|l|l|}
\hline \multicolumn{1}{|c|}{$\begin{array}{c}\text { Processo } \\
\text { Cognitivo }\end{array}$} & \multicolumn{1}{c|}{ Implicaçōes de Design } \\
\hline Atenção & $\begin{array}{l}\text { A informação precisa ficar visível quando for necessário e deve-se evitar } \\
\text { grande quantidade de informações na interface. }\end{array}$ \\
\hline Percepção & $\begin{array}{l}\text { Utilizar ícones que possibilitem ao usuário descobrir rapidamente seu } \\
\text { significado, usar sons claros e usar textos legíveis. }\end{array}$ \\
\hline Memória & $\begin{array}{l}\text { Inserir funcionalidades que possibilitem aos usuários reconhecer } \\
\text { determinada funcionalidade sem esforços }\end{array}$ \\
\hline Aprendizagem & $\begin{array}{l}\text { Criar interfaces que incentivam a exploração, além de restringir as opções } \\
\text { de modo a guiar os usuários na seleção das ações mais adequadas. }\end{array}$ \\
\hline
\end{tabular}


VIII Congresso Brasileiro de Informática na Educação (CBIE 2019)

Anais do XXX Simpósio Brasileiro de Informática na Educação (SBIE 2019)

\section{Trabalhos Relacionados}

Campos (2017) define padrões de usabilidade em sites tendo como público as crianças em fase de alfabetização com o objetivo de realizar o redesign de um site voltado para esse público-alvo. Para isto, foi realizado uma avaliação das interfaces com crianças na faixa etária de 6 a 8 anos no intuito de levantar dados de usabilidade para o desenvolvimento do projeto. Como resultados, o redesign proposto mostrou-se mais eficiente em comparação com o site existente, pois as crianças se sentiam muito confusas nesse dado à grande quantidade informações em tela e pela falta de um grid hierárquico. Além disso, o fato das telas serem muito extensas também foi um ponto identificado por prejudicar a navegação dos usuários.

O estudo de Batista et al. (2017) relata o design do processo de atualização de um repositório de objetos de aprendizagem para Matemática. Como resultados, os autores realizaram o levantamento de requisitos usando a linguagem Unified Modeling Language (UML) por meio dos diagramas de casos de uso e de classes, fizeram os protótipos de telas para a interação do usuário com o sistema e as ferramentas de desenvolvimento selecionadas para a implementação do repositório do OBAMA 2.0. A pesquisa de Vilar et al. (2019) investiga como a tecnologia pode auxiliar a aprendizagem colaborativa entre crianças de 7-11 anos de idade que possuem habilidades visuais distintas. Para tanto, exploram o Torino, uma linguagem de programação física para ensinar construções de programação e pensamento computacional. Semelhante a ideia do Scratch, para criação dos algoritmos, as crianças conectam e manipulam objetos táteis para criar música, histórias em áudio ou poesia. Como resultados, apontam a efetiva participação de 30 professores sem especialidade na área da proposta que aprenderam fazendo o experimento e 75 crianças no Reino Unido em três meses.

Neste sentido, este artigo é similar ao trabalho de Campos (2017), pois insere e discute a importância dos critérios de IHC no redesign do projeto, diferenciando-se pela aplicação e por não fazer uso de teste de usabilidade, mas, sim, revisa e identifica as lacunas existentes nos artigos Andrade, Medeiros and Medeiros (2017), Medeiros et. al (2018a), Medeiros et. al (2018b) sobre Poesia Compilada já publicados. Em Batista et al. (2017) se assemelha por propor o redesign de um sistema para uso educacional, entretanto, traz outras contribuições por apresentar o sistema já em desenvolvimento e não somente os protótipos de telas. Em relação ao estudo de Vilar et al. (2019), assemelha-se por propor uma plataforma para auxiliar no ensino de PC acessível para pessoas com deficiência visual baixa visão, tendo como público-alvo alunos do Ensino Fundamental, entretanto, distingue-se, pois, a proposta deste estudo tem como propósito ser acessível financeiramente e estar disponível na internet para ser acessado sempre que necessário, sem custo para os participantes.

Além destes referenciais, para melhor conhecimento, foi realizada uma busca nas bases Google Scholar, ACM, CEIE e RENOTE no período de 2015-2019, contudo, não foi localizado nenhum outro estudo que vem ao encontro do objetivo deste artigo, assim, este trabalho é inovador por propor atuar com o público-alvo alunos do Ensino Fundamental, e adotar o gênero textual poema através do Manifesto Poesia Compilada em um único ambiente web acessível para deficientes visuais baixa visão. 
VIII Congresso Brasileiro de Informática na Educação (CBIE 2019)

Anais do XXX Simpósio Brasileiro de Informática na Educação (SBIE 2019)

\section{Metodologia}

O estudo foi dividido em três fases: i) estudar e analisar princípios e heurísticas de usabilidade; ii) analisar a antiga interface e iii) propor redesign da plataforma. Neste sentido, a primeira etapa se concentrou em compreender os conjuntos de princípios, diretrizes (guidelines) e heurísticas que representam objetivos gerais e padrões Mayhew, (1999). Os conjuntos mais conhecidos de princípios e diretrizes são os de Norman (1988), de Nielsen (1993) e as regras de ouro de Shneiderman (1998). Destaca-se que tais diretrizes são de grande relevância em todas as etapas do projeto de um sistema interativo, tendo em vista que ajudam a nortear tanto a construção quanto a avaliação da interface proposta.

Em seguida, foi analisado o que já havia publicado acerca do projeto Poesia Compilada, como resultados da primeira ação, encontrou-se pesquisas de (Andrade, Medeiros and Medeiros (2017), Medeiros et. al (2018a), Medeiros et. al (2018b) sobre Poesia Compilada já publicados. Em Batista et al. (2017). E nesse sentido, percebeu-se que estudos citam a aplicação das diretrizes do WCAG $2.0^{1}$ ou apontam para o redesign de interface daquelas que já foram produzidas tomando como base os critérios de IHC Do Rêgo, Garrido and Mattos (2018). Observou-se, também, que elas apontam, de maneira geral, não especificando bem qual a necessidade a ser aplicada.

Com base nos resultados e consequente conhecimento adquirido por meio da etapa inicial, analisou-se a interface da primeira versão da plataforma Poesia Compilada. Nela, entendeu-se que o site e o editor Poesia() apresentavam ações que os distanciavam um do outro e não permitiam compreendê-los como um só. Por conseguinte, percebeu-se que o sistema não apresentava documentação, nem dispunha de critérios de IHC, tais como comunicabilidade, usabilidade e acessibilidade. Em busca de propor soluções, a terceira etapa iniciou-se com a identificação do que seria necessário realizar em uma nova elicitação de requisitos e o redesign da nova plataforma, conforme essa elicitação, a fim de melhorar a interação com o usuário, a relevância pedagógica e construir uma documentação. Nas seções a seguir, serão abordadas as telas do antigo site que comportava o editor e serão apresentados os requisitos que levaram à construção da nova plataforma e seu editor, para então, expor o redesign que gerou as novas telas, as quais, apesar de fazerem parte de um único sistema, serão exploradas separadamente para que se proporcione melhor compreensão sobre a aplicação dos aspectos de IHC em sua interface.

\subsection{Interfaces do site Poesia Compilada e editor Poesia}

A Figura 1(a) dispõe sobre a tela inicial do site Poesia Compilada e, nela, é possível observar que não existe a disposição de um menu, apenas contém um botão que leva ao blog do site e em seu corpo disponibiliza o Manifesto Literário Poesia Compilada.

1 WORLD WIDE WEB CONSORTIUM et al. Web content accessibility guidelines (WCAG) 2.0. 2008. 
VIII Congresso Brasileiro de Informática na Educação (CBIE 2019)

Anais do XXX Simpósio Brasileiro de Informática na Educação (SBIE 2019)

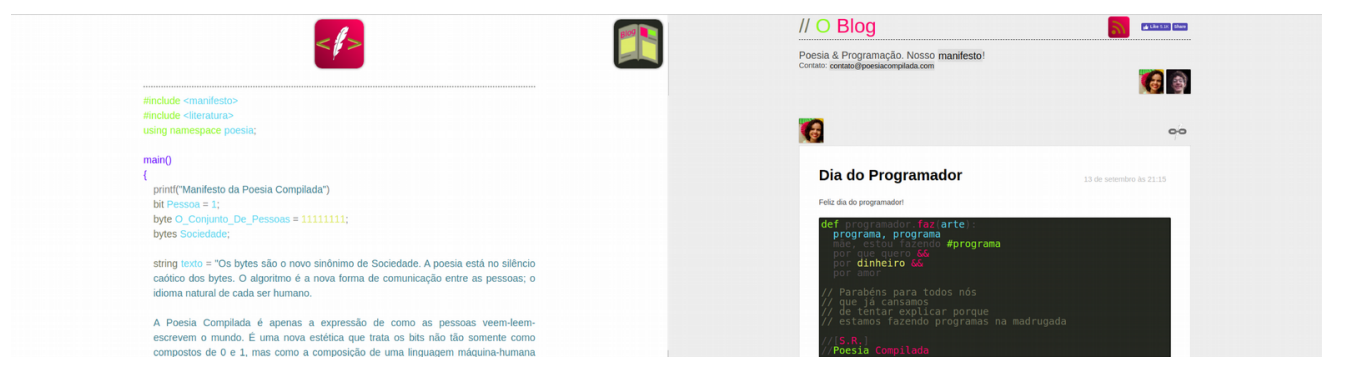

Figura 1(a): Tela inicial do Manifesto Figura 1(b): Tela do blog do Manifesto. Fonte: poesiacompilada.com Fonte: poesiacompilada.com/blog/index.html

Na Figura 1(b), encontra-se o blog do Manifesto e em uma breve consulta, ele apresenta conteúdos sobre Poesias Compiladas, poesias que são escritas utilizando a junção da sintaxe do poema com a sintaxe de algoritmo, as quais foram criadas e enviadas pelo público em geral por meio de um e-mail disponível logo abaixo do nome "O Blog", bem como apresenta fotos dos autores do Manifesto, o símbolo de link, que permite o compartilhamento somente daquela poesia, e os botões para compartilhar ou curtir na rede social Facebook. Nela, não existe um botão para voltar para o manifesto, sendo necessário sair da leitura do Blog. A Figura 2 apresenta a interface do editor Poesia, um dos grandes problemas é que só se consegue acessá-lo por meio do endereço poesiacompilada/editor, não existindo um ícone na tela inicial do Manifesto e também no blog que o leve para essa página. Bem como, as cores sugeridas só aparecem dessa forma no navegador Mozilla Firefox, sendo a navegação no Chrome e demais navegadores prejudicadas por esse erro e pelo fato de não terem como salvar as poesias. Um outro problema geral do editor é o botão de ajuda, pois, apesar dele existir, quando clicado não apresenta nenhum conteúdo.

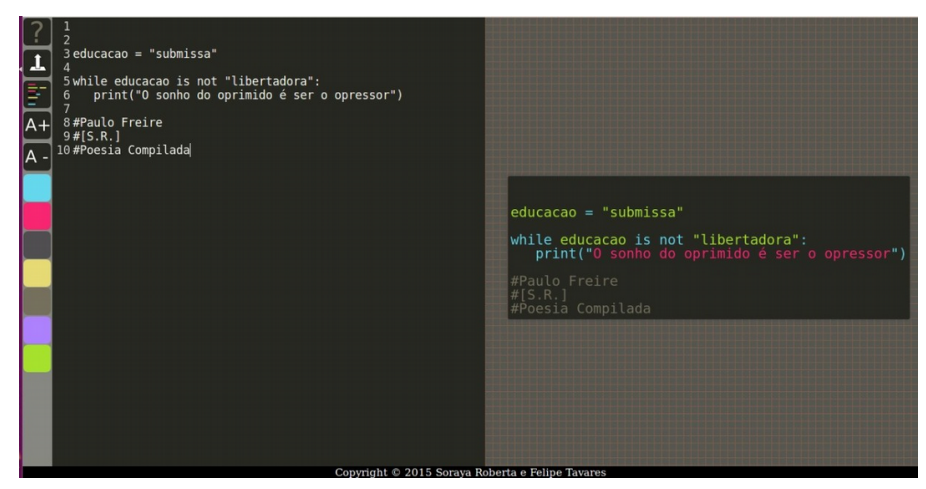

Figura 2. Antiga interface do editor Poesia. Fonte: Autor.

\subsection{Especificação de Requisitos}

Em consonância com o proposto na metodologia, neste espaço serão apresentados os Requisitos Funcionais e Não Funcionais, por questões de otimização de espaço e delimitação do objetivo, eles serão dispostos de maneira sucinta. Além disso, os requisitos aqui apresentados também estão explorados nas próximas seções através da apresentação das telas do sistema após a refatoração. Neste sentido, destaca-se que o principal ponto do redesign foi a refatoração dos requisitos não funcionais, usabilidade, responsividade e acessibilidade, destacando-se como principais fatores apontados como 
dificuldade na utilização da plataforma. Mas, também foram acrescentados os requisitos funcionais, tais como inserir um menu na tela inicial com todas as seções disponíveis pelo sistema, a inserção de um botão de login, que permite demarcar o acesso ao editor, a existência de botões de contraste e tamanho da fonte tanto na tela inicial da plataforma quanto no editor, cabendo destacar que nele foi implementado dos dois lados, além disso, suas paletas de cores para alterar a cor da poesia, bem como os seus botões de contraste e salvar.

\section{3. (Re)design das telas}

Para que se cumpra com os requisitos levantados, foi desenvolvido a plataforma Poesia Compilada que comporta o editor Poesia(). Neste sentido, quando, no decorrer das próximas seções, for utilizada a expressão Plataforma, refere-se a todo o site que comporta o editor Poesia(). Na plataforma o usuário realiza dentre outras ações o seu cadastro para ter acesso ao editor Poesia() e ao logar-se nele, pode escrever poesia compiladas. A área administrativa é reservada para os desenvolvedores do sistema editarem informações sobre o projeto. As telas e demais especificações, encontram-se dispostas nas seções, a seguir.

\subsection{Interface da Plataforma Poesia Compilada}

A plataforma, apresentada na Figura 3, é composta por um menu superior que contém os campos Sobre, em que é abordado o histórico e os desdobramentos da pesquisa para sua elaboração, seguido de um campo acerca da equipe, em que a equipe de desenvolvimento e os coordenadores são descritos; Manifesto, onde se localiza o Manifesto Literário que deu origem aos estudos relacionais entre PC e o gênero textual poema; Publicações, em que é possível encontrar os trabalhos científicos publicados do projeto com seus respectivos resumos e links para downloads e, por último o botão, Entrar, que permite o acesso para o editor Poesia().

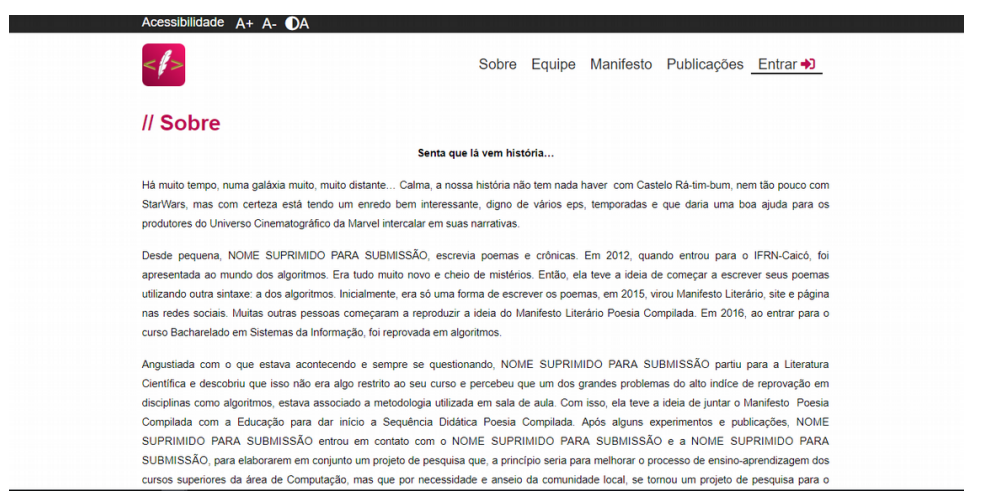

Figura 3. Tela inicial da plataforma. Fonte: Autor.

Ao lado da seção "sobre" do menu, encontra-se a Logo do projeto, e em cima de ambos, a seção de acessibilidade com os botões para aumentar, diminuir e mudar o contraste da página. Toda a plataforma apresenta o critério de comunicabilidade, em que cada elemento presente possui uma identificação sobre sua utilidade. Para permitir que usuários com baixa visão tenham acesso, a plataforma utiliza o leitor de tela, que permite além da comunicabilidade, que ele escute a descrição ao arrastar o cursor do 
VIII Congresso Brasileiro de Informática na Educação (CBIE 2019)

Anais do XXX Simpósio Brasileiro de Informática na Educação (SBIE 2019)

mouse sobre botões ou demais conteúdos das telas.

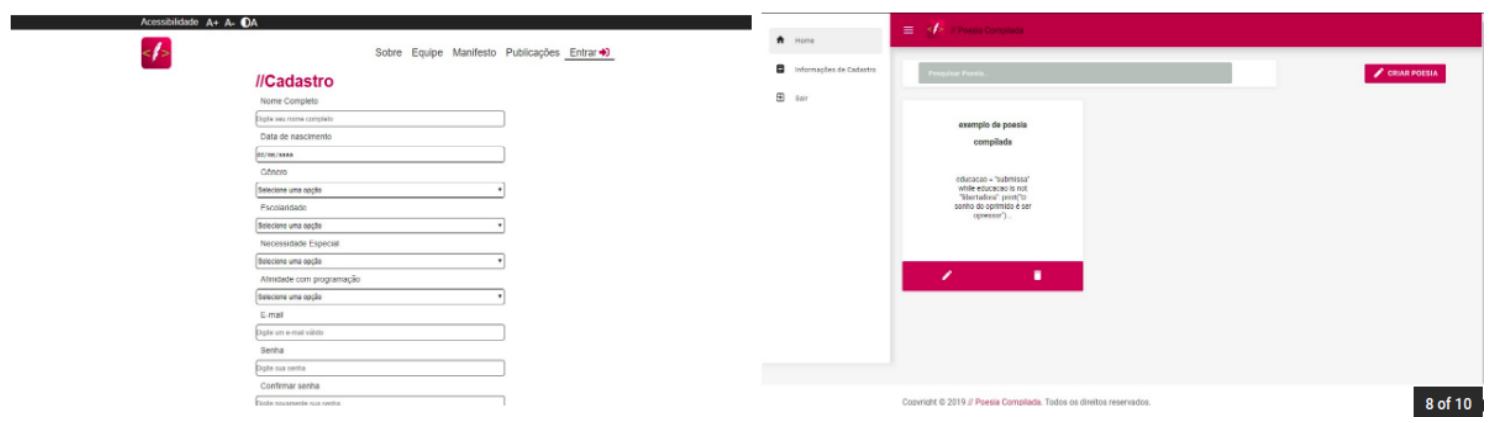

Figura 4(a). Tela de Cadastro de usuário Figura 4(b). Repositório de poesias compiladas. Fonte: Autor

De maneira geral, uma das diferenças apresentadas como melhorias por esse processo de redesign é o cadastro do usuário, disposto na Figura 4, o qual gera a possibilidade de conhecê-los, permitindo criar dados que irão viabilizar análises sobre o público-alvo, a exemplo da identificação de necessidades física, motora ou psicológica que precisem ser atendida pelo sistema. Por causa disso, o usuário pode criar, editar, excluir, salvar e exportar suas poesias, tendo a plataforma como seu repositório de poemas compilados. Além disso, a presença da comunicabilidade que permite que o usuário deficiente visual baixa-visão, consiga ter acesso a plataforma, bem como um espaço que atua como um repositório, em que é possível ver as poesias que foram feitas e salvas, bem como editálas ou excluí-las. Por questão de otimização de espaço, a visualização delas tiveram que ser reduzida.

\subsection{Interface do Editor Poesia()}

O editor Poesia () dispõe de dois espaços referenciados aqui como cadernos da esquerda e da direita. Aquele é o local em que o usuário escreve a poesia, e este, o espaço onde a poesia é exibida para ser editada e salva posteriormente. No caderno esquerdo existem os elementos referentes a "ajuda", uma outra função estabelecida pela IHC, logo abaixo, as opções de aumentar ou diminuir a exibição do caderno, bem como, botões que remetem ao contraste diurno e noturno em referência ao preto e branco da tela. Já no caderno da direita é possível escolher, na paleta de cores, uma cor para inserir em todo o poema ou em um trecho dele, bem como aumentar ou diminuir o zoom deste espaço. Acima dos cadernos existem uma caixa com a opção "título da poesia", que pode ser utilizada ao se salvar a poesia em formato png. Caso o usuário não queira inseri-lo, por default, o texto "Exemplo de poesia compilada" é adicionado. Assim como na home e demais seções da plataforma, existem a comunicabilidade e o leitor de tela, também ocorre no editor. Nele, é possível que o usuário possa selecionar trechos do poema que esteja escrevendo, para que se realize a leitura do conteúdo. A Figura 5 apresenta uma Poesia Compilada feita no editor utilizando algumas das funções descritas acima.

\section{Contribuições esperadas}

Neste trabalho foi possível discutir sobre a importância da usabilidade dos digitais em contextos educacionais e a consequente importância de um projeto de redesign para 
VIII Congresso Brasileiro de Informática na Educação (CBIE 2019)

Anais do XXX Simpósio Brasileiro de Informática na Educação (SBIE 2019)

restringir ou eliminar problemas que venham a interferir na prática pedagógica da ferramenta que esteja em utilização, através da implementação dos conceitos desenvolvidos pela IHC. Como aplicação direta, foi proposto o redesign da plat aforma Poesia Compilada. A plataforma é compreendida como um espaço lúdico que contém o editor Poesia(), que, por sua vez, auxilia no ensino de Pensamento Computacional por meio da construção de poesias compiladas através do uso do editor Poesia, uma vez que colabora na interpretação de textos, no Pensamento Algoritmo e a desenvolver a Criatividade. Tendo em vista a importância de avaliar a conformidade da interface com os padrões e heurísticas propostas pela IHC, bem como a necessidade de verificar se a mesma atende as necessidades cognitivas e pedagógicas dos usuários, destaca-se que, como trabalhos futuros, serão avaliadas tais dimensões sob diferentes aspectos.

\section{Referências}

Araujo, A. L., Andrade, W., and Guerrero, D. (2016). Um mapeamento sistemático sobre a avaliação do pensamento computacional no brasil. In Anais dos Workshops do Congresso Brasileiro de Informática na Educação, volume 5, page 1147.

Barbosa, S. and Silva, B. (2010). Interação humano-computador. Elsevier Brasil.

Barros, R. P. (2017). Cardbot: tecnologia educacional assistiva para inclusão de cddeficientes visuais na robótica educacional.

Batista, S. D., da Costa Brito1, D. T., de Melo, E. M., de Oliveira, A. M. D., de Oliveira, N. I., and Leite, D. (2017). Reconstrução de um repositório de objetos de aprendizagem para matemática. In Congresso sobre Tecnologias na Educação (Ctrl+ E).

Bell, T., Witten, I. H., Fellows, M., Adams, R., and McKenzie, J. (2011). Ensinando ciência da computação sem o uso do computador. Computer Science Unplugged ORG.

Campos, A. A. (2017). Redesign de site educacional para crianças em fase de alfabetização. B.S. thesis, Universidade Tecnológica Federal do Paraná.

Carvalho, V. and Duduchi, M. (2009). A importância da usabilidade em ambientes de educação a distância. In IV Workshop de Pós-graduação e Pesquisa do Centro Paula Souza.

Da Silva, G. T., de Souza, J. L., and Da Silva, L. A. M. (2016). Aplicação da ferramenta scratch para o aprendizado de programação no ensino fundamental i. In Anais dos Workshops do Congresso Brasileiro de Informática na Educação, volume 5, page 1285 .

De Andrade, A. P. V., Medeiros, I. G., and Medeiros, S. R. D. S. (2017). Ensino de algoritmos com poesia compilada: experiências em turmas iniciais no bacharelado em sistemas de informação. In II Congresso de Tecnologia na Educação - Ctrl+E, page 523.

Do Rêgo, B. B., Garrido, F. A., and de Souza Matos, E. (2018). Moodle como ambiente mooc: orientações para o redesign de interação. RENOTE, 16(1).

Matos, E. S. (2013). Dialética da Interação Humano-Computador: tratamento didático 
VIII Congresso Brasileiro de Informática na Educação (CBIE 2019)

Anais do XXX Simpósio Brasileiro de Informática na Educação (SBIE 2019)

do diálogo midiatizado. PhD thesis, Universidade de São Paulo.

Medeiros, S., Rabelo, H., Garcia, T. C. M., Nunes, I., Medeiros, F., Medeiros, A., and de Souza Rabelo, D. S. (2018a). Ensino de algoritmos através de poesia compilada e computação desplugada: Relato de experiência com alunos de ensino fundamental. In Anais do Workshop de Informática na Escola, volume 24, page 381.

Medeiros, S., Rabelo, H., Nunes, I., Garcia, T. C. M., Medeiros, F., de Souza Rabelo, D. S., and Medeiros, A. (2018b). Ensino de algoritmos com poemas através do editor poesia (): Relato de experiência. In Anais dos Workshops do Congresso Brasileiro de Informática na Educação, volume 7, page 341.

Medeiros, S. R. D. S. and Rabelo, H. (2018). Ensino de programação de computadores para difusão do pensamento computacional através de poesia compilada. XXIX CICT Congresso de Iniciação Científica.

Moita, F., Henrique, L., Candido, V., and Medeiros, F. M. (2017). Design e desenvolvimento de um game assistivo para autistas. In Brazilian Symposium on Computers in Education (Simpósio Brasileiro de Informática na Educação-SBIE), volume 28, page 1057.

Ortiz, J. S. B. and Pereira, R. (2018). Um mapeamento sistemático sobre as iniciativas para promover o pensamento computacional. In Brazilian Symposium on Computers in Education (Simpósio Brasileiro de Informática na Educação-SBIE), volume 29, page 1093.

Rosa, J. and Matos, E. (2015). Considerando aspectos culturais no (re) design da interação de ambientes virtuais de aprendizagem. In Brazilian Symposium on Computers in Education (Simpósio Brasileiro de Informática na Educação-SBIE), volume 26, page 852 .

Sharp, H., Rogers, Y., and Preece, J. (2005). Design de interação: além da interação homem-computador. Artmed.

Vieira, H. and Baranauskas, M. C. C. (2003). Design e avaliação de interfaces humanocomputador. Creative Commons, Brasil.

Villar, N., Morrison, C., Cletheroe, D., Regan, T., Thieme, A., and Saul, G. (2019). Physical programming for blind and low vision children at scale. In Extended Abstracts of the 2019 CHI Conference on Human Factors in Computing Systems, page INT003. ACM.

Wing, J. M. (2006). Computational thinking. Communications of the ACM, 49(3):3335 .

Mayhew, D. J. and Mayhew, D. (1999).The usability engineering lifecycle: a practitioner's handbook for user interface design. Morgan Kaufmann.

Norman, D. A. (1988).The psychology of everyday things.Basic books.

Nielsen, J. (1992). Finding usability problems through heuristic evaluation. In Proceedings of the SIGCHI conference on Human factors in computing systems, pages373-380. ACM. 